\title{
Heterozygosity of maternal factor V GI69IA (Leiden) and relationship with times of pregnancy loss among unexplained recurrent pregnancy loss
}

\section{women}

\begin{abstract}
Background: Recurrent pregnancy loss is defined by the consecutive loss of two or more pregnancies with the same partner. Recurrent pregnancy loss (RPL) or recurrent miscarriage (RM) affects from $1-5 \%$ of the reproductive age couples. This diagnosis is both emotionally challenging and confusing for most couples, as the definitive diagnosis using conventional evaluations is found in fewer than half of the couples experiencing repeated loss. The aim of this study was to evaluate the inherited heterozygosity of factor five Leiden (FVL) G1691A and Its relation to the time of recurrent spontaneous pregnancy loss.
\end{abstract}

Materials and methods: Retrospective case- control study, in which women with RPL were compared to healthy women without any evidence of spontaneous abortion. This study was undertaken at Omdurman maternity hospital in Khartoum state, Sudan. The case group consisted of one hundred women who experienced at least three or more consecutive recurrent spontaneous pregnancy loss that occurred before 20 weeks of gestation and the control group consisted of ninety five healthy women without any history of adverse pregnancy outcome.Questionnaire and direct interview were used to collect information. Genotyping was based on polymerase chain reaction. Data were entered and analyzed by SPSS program version 17.0

Result: Heterozygosity for FVL alleles G/A was $8.0 \%$ in all cases and $6.4 \%$ was found in control group. Related to association with time of recurrent pregnancy loss our result shows three times $(37.5 \%)$, four times $(50.0 \%)$ and five times $(12.5 \%)$.

Conclusion: Heterozygosity of FV Leiden G1691A could be one reason for recurrent pregnancy loss and pregnancy complications among women with unexplained pregnancy loss. Our study showed that there is an association between heterozygosity for FVL G1691A and time of recurrent pregnancy loss.

Keywords: factor V leiden, G1691A, pregnancy loss, heterozygosity, RPL, protein $\mathrm{C}$
Volume 6 Issue 5 - 2018

\author{
Asaad MA Babker,' Fath Elrahman Mahdi \\ Hassan Gameel, ${ }^{2}$ Salaheldein G Elzaki ${ }^{3}$ \\ 'Department of Medical Laboratories Science, Gulf Medical \\ University, UAE \\ ${ }^{2}$ College of applied medical science, Imam Abdulrahman Bin \\ Faisal University, Saudi Arabia \\ ${ }^{3}$ Department of Epidemiology, Tropical Medicine Research \\ Institute, Sudan
}

\begin{abstract}
Correspondence: Asaad MA Babker, Department of Medical Laboratories Science, College of Health Science, Gulf Medical University,Ajman, UAE, Email azad.88@hotmail.com
\end{abstract}

Received: August 30, 2018 | Published: October 29, 2018

\section{Introduction}

Recurrent pregnancy loss (RPL), defined as the loss of three or more consecutive pregnancies, affects $1 \%$ of couples trying to conceive. It has been estimated that $1-2 \%$ of second-trimester pregnancies miscarry before 24 weeks of gestation. ${ }^{1}$ Recurrent pregnancy loss (RPL) is caused by various genetic and non-genetic factors. After chromosome abnormality, thrombophilia is one of the most important genetic factors that could cause RPL. Factor V Leiden and factor II G20210A mutation were the most common cause of thrombophilia in the world. ${ }^{2}$ Factor $\mathrm{V}$ is one of the essential clotting factors in the coagulation cascade. Its active form, factor Va, acts as a cofactor allowing factor $\mathrm{X}$ to stimulate the conversion of prothrombin to thrombin. Activated protein $\mathrm{C}$ is a natural anticoagulant it limits the extent of clotting by destroying factor $\mathrm{V}$ and reducing further thrombin formation. Heterozygosity for a mutation in the coagulation factor $\mathrm{V}$ gene leads to resistance to activated protein $\mathrm{C}$ and represents the most common cause of inherited thrombophilia. ${ }^{3}$ Recently several studies have suggested that FVL mutation, through the production of micro thrombosis on placental bed blood vessels, causes low placental perfusion, placental infarction, and is strongly associated with RPL and maternal and fetal complications. ${ }^{4}$ Some studies have demonstrated an association between recurrent pregnancy loss and prothrombotic states rendered by some genetic single nucleotide polymorphisms of factor V Leiden G1691A.$^{2,5-7}$ Etiology is determined in approximately $50 \%$ of couples with RPL. Most of the diagnosed etiologies include endocrine abnormalities, autoimmune disorders, uterine anomalies, and genetic factors. Still, $50 \%$ of couples have no known etiology. ${ }^{8}$ The aim of the current study was to correlate heterozygosity of maternal factor V G1691A (Leiden) and relationship with times of pregnancy loss among unexplained recurrent pregnancy loss.

\section{Materials and methods}

Ethical approval was obtained from Omdurman Maternal hospital Informed consent was obtained from all patients. Genomic DNA samples of 195 Sudanese women who recruited and followed at Omdurman Maternal hospital were screened from Aug 2012 to Dec 2014. Hundred females had a history of RPL and were considered the cases and they were compared to 95 healthy reproductive Sudanese women, as control group, who had a history of two or more successful live births. Cases and controls were tested for the FV Leiden G1691A and FII G20210A. Genomic DNA was extracted from 3-5ml of EDTA anti-coagulated blood by salting ${ }^{9}$ using Master pure DNA purification 
kit for blood GF-1 Blood Dna Extraction Kit, 50 PREPS (cat. No. GFBD-050, Vivantis Technologies Sdn. Bhd., Malaysia). A 267-basepair (bp) segment of the factor $\mathrm{V}$ gene was amplified using specific forward primer (5'TCA GGC AGG AAC AAC ACC AT-3') and reverse primer 5'GGT TAC TTC AAG GAC AAA ATA CCT GTA AAG CT-3. The amplification program was as follows: Denaturation at $94^{\circ} \mathrm{C}$ for 30 seconds, annealing at $51^{\circ} \mathrm{C}$ for 30 seconds, extension at $72^{\circ} \mathrm{C}$ for 30 seconds for 35 cycles and $72^{\circ} \mathrm{C}$ for 5 minute. ${ }^{10}$ Digestion of the amplicon was performed by with MnI1 enzyme and digestion products resolved on $2 \%$ agarose gel stained with ethidium bromide (Figure 1).

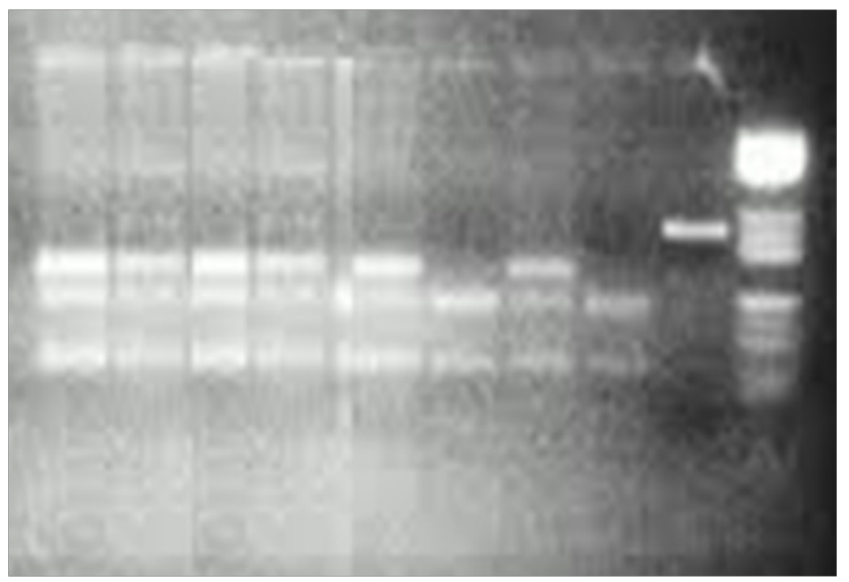

Figure I PCR amplification of FVL gene mutation:The 267bp DNA products digested with Mnll enzyme.

Lane I: molecular weight marker 50bp, lane 2: undigested PCR product (267bp); lane 3 and 5: hetrozygous mutant (AG); Lane 4, 6, 7, 8, 9 and 10:Wild type (AA)

\section{Data analysis}

Data were statistically described in terms of mean \pm standard deviation $( \pm \mathrm{SD}$ ), median and range, or frequencies (number of cases) and percentages when appropriate. Odds Ratio (OR) and the $95 \%$ confidence interval $(95 \% \mathrm{CI})$ were calculated for the presence of mutation between cases and controls and analyzed by SPSS program (version: 17.0. Data were analyzed using the Chi-square test for comparing the prevalence of MTHFR mutation between patients and controls (The test considered significant when P. value $<0.05$ ). The ethical committee approves the study; informed consent has to be obtained from participants.

\section{Results}

The participants included 195 women. Out of them, 100 had a history of 3 or more events of recurrent fetal loss (abortion, miscarriage or still birth); there was $25 \pm 4$ years and 95 women were healthy; their age was $30 \pm 4$ years. Heterozygosity of maternal factor $\mathrm{V}$ G1691A mutation distribution showed higher prevalence among cases than controls group. The mutation was detected in 8 out of 100 cases $(8.0 \%)$ and in 6 out of 94 controls $(6.4 \%) ; \mathrm{P}=0.66$, Odds Ratio $=1.28$, $95 \%$ CI (0.42 to 3.84) . Homozygous (G/G) among cases was $92 \%$ but in controls it was $93.6 \%$. G allele occurred with a frequency of $96.0 \%$ among cases and $96.8 \%$ in controls while mutant allele (A) was seen only in $4 \%$ of the cases and $3.2 \%$ of controls. The difference is statistically insignificant (Table 1) (Figure 1). Heterozygosity of maternal factor V (G1691A) related to times of recurrent pregnancy loss (Table 2).
Table I Heterozygosity of maternal factor V (GI69IA) mutation among cases of recurrent pregnancy loss compared to controls

\begin{tabular}{lllll}
\hline Genotype & $\begin{array}{l}\text { Patients } \\
\text { N (\%) }\end{array}$ & $\begin{array}{l}\text { Controls } \\
\mathbf{N}(\%)\end{array}$ & P-value & OR (95\%Cl) \\
\hline $\begin{array}{l}\text { Heterozygous } \\
\text { G/A }\end{array}$ & $8(8.0)$ & $6(6.4)$ & & \\
$\begin{array}{l}\text { Normal } \\
\text { homozygous }\end{array}$ & $92(92.0)$ & $88(93.6)$ & & \\
G/G & & & & \\
G Allele & I92(96.0) & I82(96.8) & & \\
A Allele & $8(4.0)$ & $6(3.2)$ & 0.67 & $0.76(0.27$ to 2.33$)$ \\
\end{tabular}

Table 2 Heterozygosity of maternal factor $V$ (GI69IA) related to times of recurrent pregnancy loss

\begin{tabular}{lll}
\hline \multirow{2}{*}{ Times of RPL } & \multicolumn{2}{l}{ FactorV G I 69 I A heterozygous } \\
\cline { 2 - 3 } & Positive & Negative \\
\hline Twice & 0 & $8(8.8)$ \\
Three times & $3(37.5)$ & $57(62.6)$ \\
Four times & $4(50.0)$ & $\mathrm{I} 6(\mathrm{I} 7.6)$ \\
Five times & $\mathrm{I}(12.5)$ & $6(6.6)$ \\
Six times & 0 & $\mathrm{I}(\mathrm{I} . \mathrm{I})$ \\
Seven times & 0 & $\mathrm{I}(\mathrm{I} . \mathrm{I})$ \\
Eight times & 0 & $2(2.2)$ \\
$\mathrm{P}=0.66$ & &
\end{tabular}

\section{Discussion}

Most of the studies published on the association of factor V Leiden and recurrent pregnancy loss were retrospective case- control studies. Few studies in the literature were prospective case control studies; they reported the outcome of treated pregnancies only or a comparison between treated patients with untreated patients. ${ }^{11,12}$ The current study is a retrospective analytical case control study designed to investigate the relationship between heterozygosity of maternal factor $\mathrm{V}$ (G1691A) and to number of recurrent pregnancy loss. Our results agree with several studies conducted among women with unexplained pregnancy loss. ${ }^{13-15}$ However, several studies did not report a significant association between heterozygosity of maternal factor $\mathrm{V}$ (G1691A) mutation and RPL. ${ }^{16-18}$ A difference in the prevalence of the FVL mutation in individuals may be related to different ethnic backgrounds. Some studies reported that the prevalence of factor $\mathrm{V}$ Leiden mutation varies from one nation to another. FVL has a heterogeneous distribution in different human populations, a fact that may contribute to geographic and ethnic differences in the prevalence. ${ }^{19}$ Our study also evaluated the heterozygosity of factor V (G1691A) as related to times of recurrent pregnancy loss; we found $50 \%$ positive for $\mathrm{FVL}$ associated with four times loss of pregnancy followed by $37.5 \%$ associated with three times and $12.5 \%$ with five times. Our finding is supported by some studies that concluded that women with FVL mutation are two to three times more likely to have multiple (recurrent) miscarriages or pregnancy loss during the second or third trimester. ${ }^{20}$ Some studies suggest that factor V Leiden mutation may also increase the risk of other complications during pregnancy including high blood pressure, preeclampsia, slow fetal growth, and placental abruption. ${ }^{21}$ Available data suggest that Leiden variant heterozygosity is, at most, a weak contributor to recurrent or late pregnancy loss. A meta-analysis evaluating only prospective cohort studies reported a slightly increased risk of pregnancy loss in women with the Leiden variant. ${ }^{22}$ Our results show that heterozygosity of 
factor V (G1691A) is associated with no significant trend towards risk of pregnancy complications; this may be due to the low frequency of factor V (G1691A) mutation among African people. ${ }^{23}$ Unfortunately, there is still an absence of adequate data to support or confirm the role of FVL mutation among unexplained pregnancy loss. Association between factor $\mathrm{V}$ Leiden mutation and pregnancy complications has not been confirmed and most women with factor $\mathrm{V}$ Leiden thrombophilia have normal pregnancies under good management and control.

\section{Conclusion}

Inherited heterozygosity of FV Leiden G1691A could be one reason for recurrent pregnancy loss and pregnancy complications among women with unexplained pregnancy loss. Our study shows that there is an association between heterozygosity for FVL G1691A and times of recurrent pregnancy loss.

\section{Acknowledgements}

We are grateful to the patients and healthy individuals for participating in our study.

\section{Conflict of interest}

There was no conflict of interest, and the paper is not being considered by another journal. The paper was self-funded.

\section{References}

1. Jeve YB, Davies W. Evidence-based management of recurrent miscarriages. J Hum Reprod Sci. 2014;7(3):159-169.

2. Teremmahi Ardestani M, Nodushan HH, Aflatoonian A, et al. Case control study of the factor V Leiden and factor II G20210A mutation frequency in women with recurrent pregnancy loss. Iran J Reprod Med. 2013;11(1):61-64.

3. Wüthrich RP, Cicvara-Muzar S, Booy C, et al. Heterozygosity for the factor $\mathrm{V}$ Leiden (G1691A) mutation predisposes renal transplant recipients to thrombotic complications and graft loss. Transplantation. 2001;72(3):549-550.

4. Kashif S, Kashif MA, Saeed A. The association of factor V leiden mutation with recurrent pregnancy loss. J Pak Med Assoc. 2015;65(11):1169-1172.

5. Cardona H, Castañeda SA, Cardona Maya W, et al. Lack of association between recurrent pregnancy loss and inherited thrombophilia in a group of Colombian patients. Thrombosis. 2012;2012:367823.

6. Goodman CS, Coulam CB, Jeyendran RS, et al. Which thrombophilic gene mutations are risk factors for recurrent pregnancy loss. Am J Reprod Immunol. 2006;56(4):230-236.

7. Gonçalves RO, Fraga LR, Santos WV, et al. Association between the thrombophilic polymorphisms MTHFR C677T, Factor V Leiden, and prothrombin G20210A and recurrent miscarriage in Brazilian women. Genet Mol Res. 2016;15(3):1-7.
8. Grimstad F, Krieg S. Immunogenetic contributions to recurrent pregnancy loss. J Assist Reprod Genet. 2016;33(7):833-847.

9. Miller SA, Dykes DD, Polesky HF. A simple salting out procedure for extracting DNA from human nucleated cells. Nucleic Acids Res. $1988 ; 16(3): 1215$.

10. Aleman MM, Walton BL, Byrnes JR, et al. Elevated prothrombin promotes venous, but not arterial, thrombosis in mice. Arterioscler Thromb Vasc Biol. 2013;33(8):1829-1836.

11. Jivraj S, Makris M, Saravelos S, et al. Pregnancy outcome in women with factor V Leiden and recurrent miscarriage. BJOG. 2009;116(7):995-998.

12. Jivraj S, Rai R, Underwood J, et al. Genetic thrombophilic mutations among couples with recurrent miscarriage. Hum Reprod. 2006;21(5):1161-1165.

13. Mohammadi MM, Al-Halabi MG, Monem FM. Prevalence of factor V Leiden mutation and its relation with recurrent spontaneous pregnancy loss in a group of Syrian women. Middle East Fertil Soc J. 2007;12(3):179-183.

14. Fakhr-Eldeen, Badawy A, Abu AlSel B et al. Factor V Leiden G1691A and Prothrombin G20210A mutations are associated with repeated spontaneous miscarriage in Northern area of Saudi Arabia. Genet Mol Res.2017:16(4):1-8.

15. Udry S, Aranda FM, Latino JO, et al. Paternal factor V Leiden and recurrent pregnancy loss: a new concept behind fetal genetics. $J$ Thromb Haemost. 2014;12(5):666-669.

16. Poursadegh Zonouzi A, Chaparzadeh N, Ghorbian S, et al. The association between thrombophilic gene mutations and recurrent pregnancy loss. $J$ Assist Reprod Genet. 2013;30(10):1353-1359.

17. Cardona H, Castañeda SA, Cardona Maya W, et al. Lack of association between recurrent pregnancy loss and inherited thrombophilia in a group of Colombian patients. Thrombosis. 2012;2012:367823.

18. Silver RM, Saade GR, Thorsten V, et al. Factor V Leiden, prothrombin G20210A, and methylene tetrahydrofolate reductase mutations and stillbirth: the Stillbirth Collaborative Research Network . Am J Obstet Gynecol. 2016;215(4):468.e1-468.e17.

19. Franco RF, Elion J, Santos SE, et al. Heterogeneous ethnic distribution of the factor V Leiden mutation. Genet Mol Biol. 1999;22(2):143-145.

20. Hyde KJ, Schust DJ. Genetic considerations in recurrent pregnancy loss. Cold Spring Harb Perspect Med. 2015;5(3):a023119.

21. Nurk E, Tell GS, Refsum H, et al. Factor V Leiden, pregnancy complications and adverse outcomes: The Hordaland Homocysteine Study. QJM. 2006;99(5):289-298.

22. Kujovich JL. Factor v Leiden thrombophilia. Genetics in Medicine. 2011;13:1-6.

23. Limdi NA, Beasley TM, Allison DB, et al. Racial differences in the prevalence of Factor $\mathrm{V}$ Leiden mutation among patients on chronic warfarin therapy. Blood Cells Mol Dis. 2006;37(2):100-106. 